\title{
Integração Acadêmica e Multiprofissional no PET-Saúde: Experiências e Desafios
}

Tarcísio Angelo de Oliveira Sobrinho*, Carla Patrícia Perpétua Medeiros**, Mariana Ribeiro Maia***, Tatiana Carvalho Reis****, Leonardo de Paula Miranda*****, Patrícia Ferreira Costa******

* Acadêmico do curso de graduação de medicina da Universidade Estadual de Montes Claros, MG - UNIMONTES. Voluntário do Programa de Educação pelo Trabalho para a Saúde - PET-Saúde

** Acadêmica do curso de graduação de medicina da UNIMONTES. Bolsista do PET-Saúde

*** Acadêmica do curso de graduação de ciências biológicas da UNIMONTES. Voluntária do PET-Saúde

***** Acadêmica do curso de graduação de enfermagem da UNIMONTES. Voluntária do PET-Saúde

****** Cirurgião-dentista da Secretaria Municipal de Saúde de Montes Claros, Minas Gerais. Preceptor do PET-Saúde

****** Enfermeira da Secretaria Municipal de Saúde de Montes Claros, Minas Gerais. Preceptora do PET-Saúde

\section{RESUMO}

O objetivo deste estudo foi descrever as experiências da equipe de trabalho do PET-Saúde desenvolvidas na Unidade Básica de Saúde Independência I. Trata-se de um relato de experiência retrospectivo, descritivo e inovador por possibilitar transcorrer sobre o tema da integração acadêmica e multiprofissional no PET-Saúde. As atividades foram desenvolvidas pelos acadêmicos e preceptores dos cursos de ciências biológicas, educação física, enfermagem, medicina e odontologia. O trabalho multidisciplinar visou ao atendimento integral, que foi ofertado por meio de serviços de promoção da saúde e prevenção de doenças, com vistas à melhoria da qualidade de vida dos usuários. Dentre as ações realizadas, destacam-se a realização interdisciplinar de grupos de educação em saúde, visitas domiciliares e atendimento clínico. Como desafios, a equipe encontrou a incompatibilidade curricular e a falta de um projeto político-pedagógico consistente que favoreça a atuação multiprofissional. Para superar essas dificuldades, foram realizados planejamentos prévios das ações a serem desenvolvidas, assim como a correspondência de horários disponíveis, a fim de a equi- pe melhor se preparar para execução das atividades, realizando a troca de experiências e compartilhamento do conhecimento acadêmico. Conclui-se que apesar dos avanços na construção da formação multiprofissional, os desafios só serão superados após plena integração entre os cursos das ciências da saúde de modo a permitir a maior compatibilidade e flexibilidade curricular.

\section{DESCRITORES}

Programa Saúde da Família. Educação Superior. Sistema Único de Saúde.

$\mathbf{0}$ Programa Nacional de Reorientação da Formação Profissional em Saúde (Pró-Saúde) foi criado tendo como objetivo a integração ensino-serviço e a reorientação da formação profissional, assegurando uma abordagem integral do processo saúde-doença com ênfase na Atenção Básica e promovendo transformações na prestação de serviços à população. ${ }^{1}$

Entretanto, a formação universitária ainda está centrada numa visão fragmentária no campo da disciplinaridade, fator que dificulta a formação de profissionais que saibam articular-se com outras áreas da 
saúde e que conheçam a realidade das comunidades atendidas pela Estratégia Saúde da Família (ESF). ${ }^{2}$ Essa realidade direciona para a necessidade de uma reorientação curricular efetiva e integrada entre os cursos de saúde. ${ }^{3}$

Como não é possível mudar o paradigma sanitário e o sistema de saúde sem atuar na formação dos profissionais, as Diretrizes Curriculares Nacionais (DCN) dos cursos da área da saúde estão buscando formar profissionais generalistas com uma visão crítica capaz de atender as necessidades sociais de saúde. ${ }^{4}$

O Programa de Educação pelo Trabalho para a Saúde (PET-Saúde) se insere como uma estratégia do Pró-Saúde, que incentiva a formação de grupos de aprendizagem tutorial no âmbito da ESF, por meio da iniciação ao trabalho multiprofissional e interdisciplinar dos estudantes dos cursos de graduação na área da saúde, constituindo-se como uma iniciativa intersetorial direcionada para o fortalecimento da integração ensino-serviço no âmbito da atenção básica. Iniciativas como as do PET-Saúde buscam atender o que é preconizado tanto nas diretrizes para a formação dos profissionais da saúde, quanto nas diretrizes para o exercício profissional no SUS. ${ }^{5}$

A união de profissionais de diversas áreas da saúde no trabalho em equipe é apontada como o elemento base para a consolidação da ESF, aprimorando relacionamentos interpessoais e articulando saberes. Essas equipes foram adotadas na ESF, justamente para que este modelo de atenção permita a articulação entre diferentes saberes na prática da assistência à saúde. ${ }^{6,7}$

No trabalho multiprofissional, ocorre a troca de experiências sob a ótica de uma abordagem integral e resolutiva, o que viabiliza o planejamento de ações de saúde mais eficazes. A construção do trabalho cooperativo apresenta-se como uma ferramenta eficaz para o fazer em grupo, porém, implica em superar muitos obstáculos. ${ }^{8}$

Como a inserção de equipes multiprofissionais na atenção primária é uma iniciativa inovadora que se configura como um desafio, estudos que discorram acerca dessa vivencia são importantes, a fim de contribuir na sua consolidação. Sendo assim, este estudo objetiva descrever as experiências, reflexões, desafios e contribuições da integração e atuação acadêmica e multiprofissional de uma equipe de trabalho do PETSaúde, vivenciadas em uma unidade da ESF.

\section{METODOLOGIA}

Trata-se de um estudo descritivo do tipo relato de experiência, o qual se configura como inovador por possibilitar transcorrer sobre o tema da integração acadêmica e multiprofissional no PET-Saúde.

As atividades foram desenvolvidas na unidade de saúde da ESF Independência I, situada na cidade de Montes Claros, Minas Gerais, durante o período compreendido entre Abril de 2010 e Maio de 2011, sob a coordenação de três preceptores (um dentista, uma enfermeira e uma médica, todos pertencentes à referida equipe de saúde) e atuação de acadêmicos de diferentes períodos dos seguintes cursos da Universidade Estadual de Montes Claros - UNIMONTES:

- ciências biológicas,

- educação física,

- enfermagem,

- medicina e

- odontologia.

Houve ainda o envolvimento de agentes comunitários de saúde na realização de algumas atividades.

As ações promovidas se inseriram na própria dinâmica de funcionamento da ESF e contemplaram um público diversificado, englobando as várias fases do ciclo de vida, a saber:

- crianças,

- gestantes,

- adolescentes,

- adultos,

- idosos,

- hipertensos e diabéticos,

- todos usuários adscritos no território da unidade de saúde abordada.

É importante ressaltar que houve a realização prévia de grupos de discussão acerca de temas relevantes relacionados à prática, destacando-se os princípios da ESF, territorialização e andragogia, assim como ocorreu o planejamento conjunto das atividades a serem desenvolvidas. Os grupos promovidos se embasaram na busca da vivência cotidiana do usuário e as atividades clínicas resgataram o conhecimento científico adquirido na academia, sempre buscando acrescentar mais aprendizado ao estudante, por meio de sua inserção no cenário de prática da atenção primária em saúde.

\section{RESULTADOS}

O trabalho multidisciplinar realizado nesse período visou ao atendimento integral, que foi ofertado por meio de serviços de promoção da saúde e prevenção de doenças, com vistas à melhoria da qualidade de vida dos usuários. Dentre as ações realizadas, des- 
tacam-se a realização interdisciplinar de grupos de educação em saúde, visitas domiciliares e atendimento clínico.

Nas atividades de educação em saúde, foi adotada a metodologia de grupos operativos, operacionalizada por meio de encontros com participação de um número reduzido de usuários com características em comum. Os temas abordados foram escolhidos buscando contemplar a demanda do território, para grupos pré-definidos pela unidade, tais como:

- diabetes,

- hipertensão,

- planejamento familiar,

- gestação,

- idosos e

- saúde mental, com ênfase na promoção e proteção da saúde.

Com o propósito de estreitar o vínculo entre as famílias e a equipe da ESF e do PET-Saúde, foram realizadas visitas domiciliares. Estas atividades permitiram a apresentação da equipe do PET-Saúde aos usuários, bem como a proposta de realização de um planejamento integrado que envolvesse a comunidade, fortalecendo a diretriz do controle social. Exemplo satisfatório desta atuação foi o resgate dos pacientes com transtorno mental, que estavam afastados da unidade e que passaram a integrar um grupo de BemEstar em Saúde, realizado mensalmente pela equipe.

Os discentes tiveram a oportunidade de acompanhar os atendimentos clínicos realizados pelos profissionais da equipe, preceptores do PET-Saúde (médico, odontólogo e enfermeira). As práticas se constituíram de consultas de pré-natal, puerpério, puericultura, saúde da mulher e demanda espontânea. No decorrer da realização dessas atividades, houve a troca de experiências e o esclarecimento de dúvidas. Ao auxiliar os preceptores, os estudantes tiveram a possibilidade de aplicar os conhecimentos teóricos adquiridos na academia.

Como desafios, a equipe evidenciou a incompatibilidade curricular e a falta de um projeto políticopedagógico institucional consistente que favorecesse a atuação das várias classes profissionais. Para superar essas dificuldades, foi elaborado um Planejamento Estratégico Situacional das ações a serem desenvolvidas, englobando, inclusive, a correspondência dos horários de disponibilidade dos participantes envolvidos no processo, a fim de garantir à equipe uma melhor preparação e execução das atividades, tendo sempre como foco uma prática transdiciplinar.

\section{DISCUSSÃo}

Observa-se, pela literatura, que o trabalho em equipe é a base para ações integrais na saúde ${ }^{9}$ e condição necessária para atender com qualidade as necessidades dos usuários de acordo com a variabilidade de situações. ${ }^{10}$

Destaca-se que tanto os grupos operativos quanto as visitas domiciliares possibilitaram o estreitamento do vínculo com os usuários. Segundo Souza et al. $(2003)^{11}$ e Teixeira et al. (2000), ${ }^{12}$ o vínculo é conseqüência de uma relação mais próxima da população com a equipe de saúde por conseqüência das visitas domiciliares, que facilita a adesão da população ao serviço de saúde. A população sente-se melhor cuida$\mathrm{da}$, pois a equipe intervém com visão mais ampliada pelo conhecimento da população, estimulando sua autonomia e participação no tratamento, numa relação de respeito e valorização das particularidades, inclusive co-responsabilizando a população pelo seu próprio bem-estar.

O acompanhamento das atividades clinicas inserem-se como uma das estratégias do Pró-Saúde, relacionada mais especificamente ao eixo cenários de práticas, o PET-Saúde busca incentivar a interação ativa dos estudantes e docentes dos cursos de graduação em saúde com os profissionais dos serviços e com a população. Ou seja: induzir que a escola integre, durante todo o processo de ensino-aprendizagem, a orientação teórica com as práticas de atenção nos serviços públicos de saúde, em sintonia com as reais necessidades dos usuários do Sistema Único de Saúde. ${ }^{13}$

A incompatibilidade curricular e a falta de um projeto político-pedagógico para atuação das várias classes profissionais foram verificadas neste trabalho. Segundo Pedrosa et al. (2001), ${ }^{14}$ o PSF constitui-se de equipes multiprofissionais que devem atuar em uma perspectiva interdisciplinar. Os membros da equipe articulam suas práticas e saberes no enfrentamento de cada situação identificada para propor soluções conjuntamente e intervir de maneira adequada, já que todos conhecem a problemática.

\section{CONCLUSÃo}

A experiência proporcionada pelo PET-Saúde aos acadêmicos dos diversos cursos da área da saúde permite vivenciar e atuar nas unidades das ESFs, contribuindo para a interação acadêmica e atuação transdiciplinar e multiprofissional dos mesmos. Aaquisição de novos aprendizados propiciada é extremamente relevante para uma formação diferenciada, o que torna o futuro profissional mais preparado para enfren- 
tar o mercado de trabalho.

Nesse sentido, destaca-se que houve uma satisfatória integração entre os atores durante o processo de trabalho relatado, tanto no que diz respeito à relação docente-discente, quanto à interação academia e serviço. Deve-se enfocar que, apesar dos avanços alcançados com a proposta de uma atuação profissional diversificada e interdisciplinar, os desafios mencionados só deverão ser superados após a plena integração entre os cursos das ciências da saúde, de modo a permitir, dessa forma, uma maior flexibilidade e compatibilidade curricular.

\section{ABSTRACT}

Academic and Multiprofessional Integration in the PET - Health Program: Experiences and Challenges

The purpose of this study was to describe the experiences of the PET - Health staff at the Independencia I Basic Health Center. It is a retrospective, descriptive and innovative experience-based report that makes it possible to address academic and multiprofessional integration in the PET - Health program. The related activities were developed by students and instructors of Biology, Physical Education, Nursing, Medicine and Dentistry. The multidisciplinary work focused on full-time treatment offered through health promotion and diseases prevention services to increase the life quality of users. Among the actions performed, we can highlight the forming of interdisciplinary health education groups, home visits and clinical treatment. Among the challenges, the team came up against curriculum incompatibility and lack of a consistent political-pedagogical project favoring multiprofessional performance. To overcome these difficulties, prior planning of actions to be developed and matching of schedule availability was undertaken so that the team could be better prepared to perform its activities, exchange experiences and share academic knowledge. In conclusion, despite advances regarding the development of multiprofessional training, the challenges can only be overcome after the full integration of health science courses, to promote greater curriculum compatibility and flexibility.

\section{DESCRIPTORS}

Family Health Program. Education, Higher. Unified Health System.

\section{REFERÊNCIAS BIBLIOGRÁFICAS}

1. Brasil. Ministério da Saúde. Ministério da Educação. Pró-saúde: programa nacional de reorientação da formação profissional em saúde. Brasília: MS/MEC; 2009. [acesso 26 jun 2010]. Disponível em: http://www.prosaude.org/not/prosaude-maio2009/ proSaude.pdf.

2. Moretti-Pires RO. Complexidade em Saúde da Família e formação do futuro profissional de saúde. Interface (Botucatu) 2009;13(30): 153-66.

3. Moretti-Pires RO, Campos DA. Equipe multiprofissional em Saúde da Família: do documental ao empírico no interior da Amazônia. Rev Bras Educ Med [online] 2010; 34(3):379-89.

4. Silva RPG, Rodrigues RM. Sistema Único de Saúde e a graduação em enfermagem no Paraná. Rev Bras Enferm 2010; $63(1): 66-72$.

5. Brasil. Ministério da Saúde. Pró-saúde: programa nacional de reorientação da formação profissional em saúde / Ministério da Saúde, Ministério da Educação. - Brasília: Ministério da Saúde, 2005. [acesso 29 jun 2011] Disponível em: http://www. abem-educmed.org.br/pro_saude/publicacao_pro-saude.pdf.

6. Brasil. Ministério da Educação. Ministério da Saúde. Programa Nacional de Reorientação da Formação Profissional em Saúde - Pró-Saúde. Brasília; 2007.

7. Marquesa JB, Aprígiob DP, Mellob HLS, Silvab JD, Pintob LN, Machadoc DCD, et al. Contribuições da equipe multiprofissional de saúde no programa de saúde da família (PSF): uma atualização da literatura. Rev Baiana de Saúde Pública 2007; $31(2): 246-55$.

8. Ferreira RC, Varga CRR, Silva RF. Trabalho em equipe multiprofissional: a perspectiva dos residentes médicos em saúde da família. Ciênc Saúde Coletiva [online] 2011; 14(1):1421-8.

9. Santos BRL. O PSF e a enfermagem. Rev Bras Enfermagem 2000; 53(N Esp):49-53.

10. Schraiber LB, Peduzzi M, Sala A, Nemes MTB, Castanhera ERL, Kon R. Planejamento, gestão e avaliação em saúde: identificando problemas. Ciênc Saúde Coletiva 1999; 4(2):221-42.

11. Souza RA, Carvalho AM. Programa de Saúde da Família e qualidade de vida: um olhar da psicologia. Estud Psicol (Natal) 2003; 8(3):515-23.

12. Teixeira RA, Mishima SM. Perfil dos trabalhadores de enfermagem no Programa de Saúde da Família. Rev Bras Enfermagem 2000; 53(3):386-400.

13. HaddadI AE, Campos FEII, Fruet MSBFI; Sigisfredo LB; Passarella TM; Ribeiro TCV. Programa De Educação Pelo Trabalho Para A Saúde - Pet-Saúde. Cadernos ABEM 2009;5: 07.

14. Pedrosa JIS, Teles JBM. Consenso e diferenças em equipes do Programa Saúde daFamília. RevSaúde Pública 2001;35(3):30311. 